\title{
Article \\ Golf-Putting Performance in Skilled Golfers at Different Distances to the Hole
}

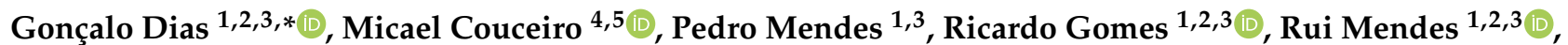 \\ Vasco Vaz ${ }^{1}$, Fernando Martins ${ }^{2,3,6} \mathbb{D}^{\text {, J José Gama }}{ }^{1}$ and Maria António Castro ${ }^{3,7}$ (D)
}

1 Research Unit for Sport and Physical Activity (CIDAF), University of Coimbra, 3004-531 Coimbra, Portugal; pmendes@esec.pt (P.M.); rimgomes@esec.pt (R.G.); rmendes@esec.pt (R.M.); vascovaz@fcdef.uc (V.V.); josemiguelgama@hotmail.com (J.G.)

2 Polytechnic Institute of Coimbra, ESEC, ASSERT, 3030-329 Coimbra, Portugal; fmlmartins@esec.pt

3 Polytechnic Institute of Coimbra, IIA, ROBOCORP, 3030-329 Coimbra, Portugal; maria.castro@ipleiria.pt

4 Ingeniarius, Ltd., Rua Nossa Senhora da Conceição 146, 4445-147 Alfena, Portugal; micael@ingeniarius.pt

Institute of Systems and Robotics (ISR), University of Coimbra (FCTUC), 3004-531 Coimbra, Portugal

6 Instituto de Telecomunicações (IT), 6201-001 Covilhã, Portugal

7 School of Health Sciences, Polytechnic of Leiria, Portugal, CEMMPRE, University of Coimbra, 3004-531 Coimbra, Portugal

* Correspondence: goncalodias@fcdef.uc.pt

Citation: Dias, G.; Couceiro, M.; Mendes, P.; Gomes, R.; Mendes, R.; Vaz, V.; Martins, F.; Gama, J.; Castro, M.A. Golf-Putting Performance in Skilled Golfers at Different Distances to the Hole. Appl. Sci. 2021, 11, 11785. https://doi.org/10.3390/ app112411785

Academic Editor: Matej Supej

Received: 19 October 2021

Accepted: 7 December 2021

Published: 11 December 2021

Publisher's Note: MDPI stays neutral with regard to jurisdictional claims in published maps and institutional affiliations.

Copyright: (c) 2021 by the authors. Licensee MDPI, Basel, Switzerland. This article is an open access article distributed under the terms and conditions of the Creative Commons Attribution (CC BY) license (https:// creativecommons.org/licenses/by/ $4.0 /)$.

\begin{abstract}
Golf putting occurs under highly variable conditions. Therefore, adapting to different putting distances is a challenge that the golfer faces and understanding the variables underlying performance in this task is important. The main objective of this study was to analyse the golf-putting performance in skilled golfers at different distances to the hole. Fourteen highly skilled golfers, adult male and right-handed ( $41.5 \pm 13.2$ years with an average handicap of $2.3 \pm 1.7)$ performed the golf putting on a natural grass green, completing a total of 120 randomly ordered trials, 30 trials at each of the four different practice conditions $(1,2,3$, and $4 \mathrm{~m}$ away from the hole). A smart engineered putter (InPutter) was used as the data acquisition instrument to record several process variables at $100 \mathrm{~Hz}$. Results indicated that golf-putting distances constrained movement organisation variables in specific ways. For example, as the distance to the hole changes, so do the informational constraints, shaping how a golfer needs to regulate performance. We concluded that the effects of different golf-putting distances required the implementation of functional solutions uniquely adjusted to each player. Furthermore, the perception that the player withdrew from the properties of the context (e.g., distance to the hole) was important to adapt the golf-putting process variables (e.g., amplitude and duration of the movement, among others). All these factors led to a decisive influence on how the golfer hit the ball and adjusted his performance. A major implication of developing a better understanding of the role of performance in golf putting is that coaches and players should allow functional movement behaviours to emerge during practice.
\end{abstract}

Keywords: task constraints; pro-golfer analysis; motor performance; wireless technology; InPutter

\section{Introduction}

Pelz [1] claimed that a golfer who participates in the PGA Tour faces several constraints, being susceptible to a high variability of practice conditions that requires constant adaptations to putting distances. Consequently, an important challenge is to understand how each golfer learns to adapt performance under different distances to the hole [2].

Based on results obtained in the USPGA golf tour putting data analysis, Suzuki et al. [3] indicated that the short putting ability is an important factor in golf performance, adding to the key skills of the golfer the control of distance and direction in the putt.

In a specific way, Suzuki et al. [3] indicated that the proportion of missed putts from a short distance in professional golfers when compared to novice golfers, as well as the comparison with putts from the nondominant stance, has not been scientifically reported in 
previous studies. For instance, professional golfers tend to present high level of lengthways putting control from their dominant stance, as a consequence of the several years of training and experience [1]. As such, professional golfers generally present a significantly lower distance error than novice golfers in both dominant and nondominant putting stance from a $2 \mathrm{~m}$ target.

On the other hand, in a study that analysed the putting stroke in professional and novice golfers [4], the peak impact force was generated at $3 \mathrm{~m}$, compared to series of putting strokes performed at a distance between $1 \mathrm{~m}$ and $3 \mathrm{~m}$.

Regarding the process variables of motor performance, Tucker et al. [5] verified that professional golfers maintained a high consistency of ball speed and the movement variability was highly individual-specific with different players adopting different performance strategies depending on the distance to the hole.

Likewise, in golf putting, the process variables (amplitude and duration of the movement, among others) are directly related to the performance of the golfer [6]. In this sense, several authors [2,7] have proposed methodologies to study the golf-putting process variables on each phase, from which particular attention has been given to the position, velocity, and acceleration of the putter.

Finally, other researchers [8,9] analysed the putting performance based on process variables, such as acceleration of limbs and body segments during movement [10]. Some of these dependent variables have been previously explored by Dias et al. 2014 [2], but a deeper understanding of the process variables (time duration, velocity, amplitude, among others) is required due to the fact that these previous experiments were carried out under of a laboratory context.

Hence, the main objective of this study was to analyse the golf-putting performance of skilled golfers at different distances to the hole in a non-laboratory scenario. We hypothesize that the manipulation of putting distance required the adoption of solutions exclusively adjusted to each player.

\section{Methods}

\subsection{Participants}

Fourteen highly skilled golfers, adult male and right-handed ( $41.5 \pm 13.2$ years with an average handicap of $2.3 \pm 1.7$ ) performed the golf putting on a natural grass green.

The athletes competed for the Portuguese Golf Federation national championship, and included the European and World Pitch and Putt Champion. Considering their availability to participate in this study $(n=14)$, we selected the golfers with lower handicaps and those who showed the best performance in the National Absolute Pitch \& Putt Championship. All participants signed an ethical consent form. The study was conducted according to the guidelines of the Declaration of Helsinki and approved by the Ethics Committee of Polytechnic Institute of Coimbra (Approval number: N145 CEIP/2021).

\subsection{Task and Apparatus}

Participants performed the golf putting on a natural grass green, completing a total of 120 randomly ordered trials, 30 trials at each of the four different practice conditions: PC1, at $1 \mathrm{~m}$ away from the hole, PC2, at $2 \mathrm{~m}$ away from the hole, PC3, at $3 \mathrm{~m}$ away from the hole and PC4, at $4 \mathrm{~m}$ away from the hole, with the putting position being adequately identified in the green [2]. A smart engineered putter, known as InPutter, was used as the data acquisition instrument to record several process variables at $100 \mathrm{~Hz}$ [11]. InPutter is a commercial solution provided by Ingeniarius [12] with the same physical properties of a common putter, with average dimensions and weight, following the regulations of both the Portuguese and International Golf Federations (Figure 1). This device maintains the representative design of golf-putting performance, without the need for any auxiliary hardware in a laboratory context as in some previous studies [11]. 


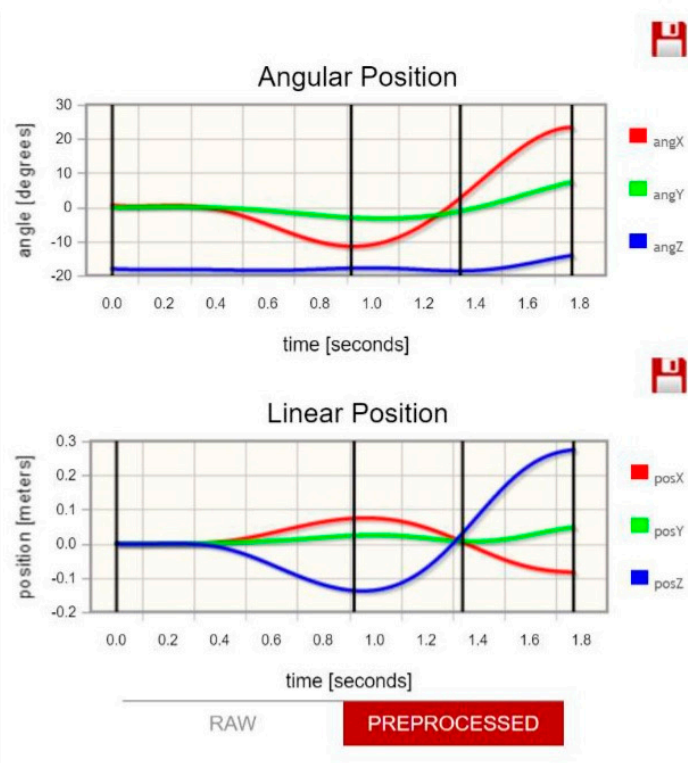



Impact Pressure [KgF.cm ${ }^{-2}$ ]

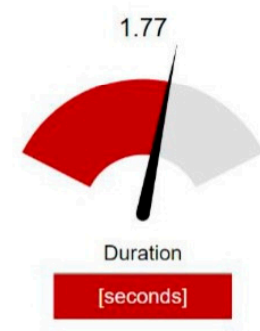

0.87

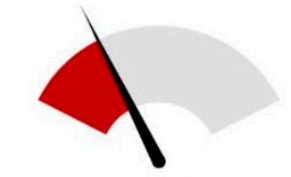

Impact Velocity [m.s $\left.\mathrm{s}^{-1}\right]$

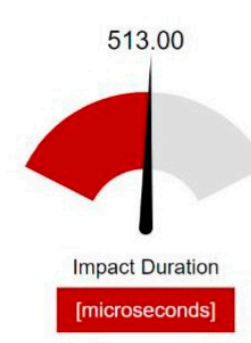

0.41

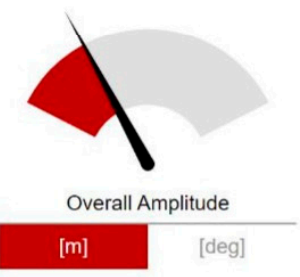

2.59



Peak Acceleration

$\left[\mathrm{m}^{\mathrm{s}} \mathrm{s}^{-2}\right] \quad\left[\mathrm{deg} . \mathrm{s}^{-2}\right]$
분

분
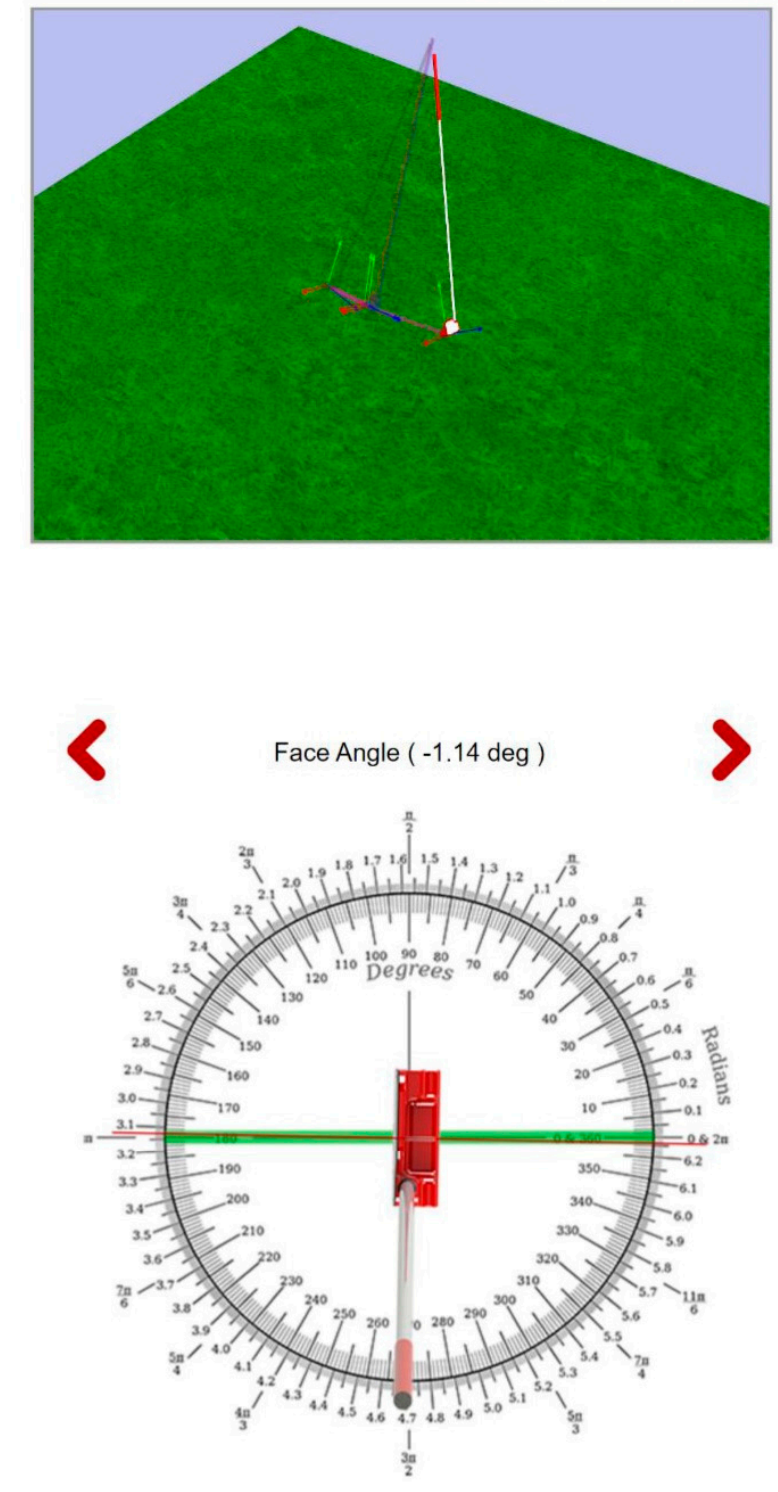

?
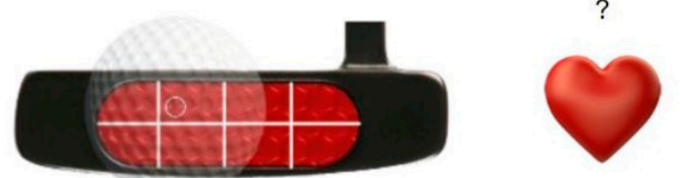

Figure 1. InPutter-data acquisition instrument. Both angular and linear (Cartesian) positions are represented for each $\mathrm{x}, \mathrm{y}$, and $\mathrm{z}$ components, following the colour scheme represented in the figure as red, green, and blue, respectively.

InPutter can compute the most relevant process variables inherent to the putting, providing them in real time and over the Internet. Data for the putting analysis comes from an IMU sensor, which includes 3D gyroscope, 3D accelerometer, and 3D magnetometer and an array of force sensors. Hence, the data likely to be obtained from InPutter are presented in Table 1. 
Table 1. Variables obtained from InPutter.

\begin{tabular}{cc}
\hline Variables Obtained from InPutter & Units \\
\hline Impact pressure & $\mathrm{KgF} . \mathrm{cm}^{-2}$ \\
Impact duration & $\mathrm{\mu s}$ \\
Movement duration in each phase & $\mathrm{s}$ \\
Amplitude of angular position & degrees \\
Amplitude of linear (cartesian) position & $\mathrm{m}$ \\
Peak angular velocity & degrees.s $^{-1}$ \\
Peak linear velocity & ${\mathrm{m} . \mathrm{s}^{-1}}^{-2}$ \\
Peak angular acceleration & degrees.s $^{-2}$ \\
Peak linear acceleration & m.s $^{-2}$ \\
Face angle & degrees \\
Declination angle & degrees \\
\hline
\end{tabular}

\subsection{Procedures}

Each participant performed three adaptation trials at a distance of $1.5 \mathrm{~m}$ from the hole. During this preliminary experiment, the players had neither verbal feedback about their movement nor about the result provided by InPutter, but they had visual access to the ball trajectory and stopping position. Putting distances and number of trials were chosen for data analysis based on previous works by Couceiro et al. [11,13]. The putting movement was analysed in four phases: (i) backswing; (ii) downswing; (iii) ball impact and (iv) follow-through. The backswing aligns the golfer's hub centre and the club head. The downswing phase starts where the backswing phase ends, and finishes immediately before the club head strikes the ball. The moment of ball impact is the time instant at which the club head strikes the ball. Lastly, the follow-through starts immediately after the point of ball impact and consists of the deceleration phase [6].

\subsection{Statistical Procedures}

The influence of practice conditions (PC1, PC2, PC3, and PC4) on all dependent variables was assessed through the one-way ANOVA test, after checking the normality and homogeneity assumptions [14]. A Scheffé post hoc was performed to make comparisons between practice conditions. When homogeneity was not verified the Games-Howell's post hoc test was used.

The estimation of the effect size, $\eta^{2}$, (proportion of the variance in the dependent variables that can be explained by the independent variables) was done according to Pallant [13]. Apart from the effect size, the power of the corresponding test was also presented. The effect size (ES) was presented as $\eta^{2}$ for one-way ANOVA test and interpreted using the follow criteria: no effect $(\mathrm{ES}<0.04)$, minimum effect $(0.04 \leq \mathrm{ES}<0.25)$, moderate effect $(0.25 \leq \mathrm{ES}<0.64)$ and strong effect $(\mathrm{ES} \geq 0.64)$ [15]. The data analysis was conducted using IBM SPSS (IBM SPSS Statistics for Windows, version 24.0. Armonk, NY: IBM Corp) software and a statistical significance of $5 \%(p<0.05)$ was defined.

\section{Results}

Table 2 presents a general overview of the results, where mean and standard deviation are shown for each Practice Condition (PC1, PC2, PC3 and PC4), along with the statistical comparisons between each $\mathrm{PC}$ for each process variable. 
Table 2. Descriptive statistics of the process variables and comparison to different Practice Conditions (PC).

\begin{tabular}{|c|c|c|c|c|c|c|c|c|c|}
\hline Process Variables & PC1 & PC2 & PC3 & PC4 & F & $p^{*}$ & $\eta 2$ & $\pi$ & ES \\
\hline $\begin{array}{c}\text { Back/downswing_amp. } \\
\text { (deg) }\end{array}$ & $9.23 \pm 2.72^{a, b, c}$ & $11.07 \pm 2.91^{\mathrm{a}, \mathrm{d}, \mathrm{e}}$ & $12.80 \pm 3.17^{b, d, f}$ & $14.78 \pm 4.27^{\mathrm{c}, \mathrm{e}, \mathrm{f}}$ & 214.33 & 0.001 & 0.28 & 1 & $\begin{array}{c}\text { Moderate } \\
\text { effect }\end{array}$ \\
\hline $\begin{array}{l}\text { Back/downswing_amp. } \\
(\mathrm{m})\end{array}$ & $0.14 \pm 0.05^{\mathrm{a}, \mathrm{b}, \mathrm{c}}$ & $0.17 \pm 0.05^{\mathrm{a}, \mathrm{d}, \mathrm{e}}$ & $0.20 \pm 0.05^{\mathrm{b}, \mathrm{d}, \mathrm{f}}$ & $0.22 \pm 0.05^{\mathrm{c}, \mathrm{e}, \mathrm{f}}$ & 218.10 & 0.001 & 0.28 & 1 & $\begin{array}{c}\text { Moderate } \\
\text { effect }\end{array}$ \\
\hline $\begin{array}{l}\text { FollowThrough_amp. } \\
\text { (deg) }\end{array}$ & $15.89 \pm 4.50^{\mathrm{a}, \mathrm{b}, \mathrm{c}}$ & $20.49 \pm 6.08^{\mathrm{a}, \mathrm{d}, \mathrm{e}}$ & $25.27 \pm 6.84^{\mathrm{b}, \mathrm{d}, \mathrm{f}}$ & $29.40 \pm 8.32^{\mathrm{c}, \mathrm{e}, \mathrm{f}}$ & 332.12 & 0.001 & 0.37 & 1 & $\begin{array}{c}\text { Moderate } \\
\text { effect }\end{array}$ \\
\hline $\begin{array}{l}\text { Followthrough_amp. } \\
\qquad(\mathrm{m})\end{array}$ & $0.29 \pm 0.07^{\mathrm{a}, \mathrm{b}, \mathrm{c}}$ & $0.35 \pm 0.09^{\mathrm{a}, \mathrm{d}, \mathrm{e}}$ & $0.43 \pm 0.11^{\mathrm{b}, \mathrm{d}, \mathrm{f}}$ & $0.48 \pm 0.11^{\mathrm{c}, \mathrm{e}, \mathrm{f}}$ & 323.87 & 0.001 & 0.37 & 1 & $\begin{array}{c}\text { Moderate } \\
\text { effect }\end{array}$ \\
\hline $\begin{array}{l}\text { Maximum_amp. } \\
\text { (deg) }\end{array}$ & $26.18 \pm 5.59^{a, b, c}$ & $32.88 \pm 6.97^{\mathrm{a}, \mathrm{d}, \mathrm{e}}$ & $39.59 \pm 8.11^{\mathrm{b}, \mathrm{d}, \mathrm{f}}$ & $45.20 \pm 8.70^{c, e, f}$ & 511.72 & 0.001 & 0.48 & 1 & $\begin{array}{l}\text { Moderate } \\
\text { effect }\end{array}$ \\
\hline Maximum_amp (m) & $0.46 \pm 0.10^{\mathrm{a}, \mathrm{b}, \mathrm{c}}$ & $0.57 \pm 0.12^{\mathrm{a}, \mathrm{d}, \mathrm{e}}$ & $0.68 \pm 0.14^{\mathrm{b}, \mathrm{d}, \mathrm{f}}$ & $0.75 \pm 0.13^{\mathrm{c}, \mathrm{e}, \mathrm{f}}$ & 450.19 & 0.001 & 0.45 & 1 & $\begin{array}{c}\text { Moderate } \\
\text { effect }\end{array}$ \\
\hline $\begin{array}{l}\text { Backswing } \\
\text { duration (ms) }\end{array}$ & $547.17 \pm 172.73^{b, c}$ & $571.50 \pm 157.19$ e & $586.31 \pm 143.73^{b}$ & $604.41 \pm 165.60^{\text {c,e }}$ & 10.51 & 0.001 & 0.02 & 0.99 & No effect \\
\hline $\begin{array}{l}\text { Downswing } \\
\text { duration (ms) }\end{array}$ & $298.21 \pm 72.87$ & $309.91 \pm 100.89$ & $300.36 \pm 82.40$ & $297.12 \pm 83.77$ & 1.95 & 0.120 & 0.01 & 0.51 & No effect \\
\hline $\begin{array}{l}\text { Impact duration } \\
\text { (ms) }\end{array}$ & $0.70 \pm 0.40$ & $0.70 \pm 0.40$ & $0.65 \pm 0.40$ & $0.65 \pm 0.40$ & 2.34 & 0.072 & 0.01 & 0.59 & No effect \\
\hline $\begin{array}{l}\text { Followthrough } \\
\text { duration (ms) }\end{array}$ & $399.76 \pm 131.47$ & $400.90 \pm 136.18$ & $421.45 \pm 144.45$ & $412.19 \pm 127.21$ & 2.43 & 0.064 & 0.01 & 0.61 & No effect \\
\hline $\begin{array}{l}\text { Maximum duration } \\
(\mathrm{ms})\end{array}$ & $1259.31 \pm 297.29^{c}$ & $1276.76 \pm 302.83$ & $1308.64 \pm 297.78$ & $1316.86 \pm 295.12^{c}$ & 3.44 & 0.016 & 0.01 & 0.77 & No effect \\
\hline $\begin{array}{l}\text { Impact velocity } \\
\left(\mathrm{m}^{\left.-\mathrm{sec}^{-1}\right)}\right.\end{array}$ & $1.11 \pm 0.16^{\mathrm{a}, \mathrm{b}, \mathrm{c}}$ & $1.39 \pm 0.18^{\mathrm{a}, \mathrm{d}, \mathrm{e}}$ & $1.69 \pm 0.25^{\mathrm{b}, \mathrm{d}, \mathrm{f}}$ & $1.90 \pm 0.27^{\mathrm{c}, \mathrm{e}, \mathrm{f}}$ & 1033.46 & 0.001 & 0.65 & 1 & $\begin{array}{l}\text { Strong } \\
\text { effect }\end{array}$ \\
\hline $\begin{array}{l}\text { Speed of impact on } \\
\left.\text { the ball (deg.sec }{ }^{-1}\right)\end{array}$ & $59.85 \pm 8.07^{a, b, c}$ & $74.31 \pm 8.34^{\mathrm{a}, \mathrm{d}, \mathrm{e}}$ & $88.01 \pm 10.61^{b, d, f}$ & $104.75 \pm 19.30^{c, e, f}$ & 997.23 & 0.001 & 0.65 & 1 & $\begin{array}{l}\text { Strong } \\
\text { effect }\end{array}$ \\
\hline $\begin{array}{l}\text { Peak Accel } \\
\left(\text { deg.sec }{ }^{-2}\right)\end{array}$ & $284.56 \pm 87.61^{\mathrm{a}, \mathrm{b}, \mathrm{c}}$ & $337.86 \pm 91.30^{\mathrm{a}, \mathrm{d}, \mathrm{e}}$ & $404.51 \pm 120.53^{\mathrm{b}, \mathrm{d}, \mathrm{f}}$ & $507.10 \pm 172.62^{c, e, f}$ & 256.07 & 0.001 & 0.31 & 1 & $\begin{array}{c}\text { Moderate } \\
\text { effect }\end{array}$ \\
\hline $\begin{array}{l}\text { Peak Accel } \\
\left(\mathrm{m} \cdot \mathrm{sec}^{-2}\right)\end{array}$ & $4.67 \pm 1.06^{\mathrm{a}, \mathrm{b}, \mathrm{c}}$ & $5.76 \pm 1.36^{\mathrm{a}, \mathrm{d}, \mathrm{e}}$ & $6.94 \pm 1.84^{\mathrm{b}, \mathrm{d}, \mathrm{f}}$ & $7.89 \pm 2.21^{\mathrm{c}, \mathrm{e}, \mathrm{f}}$ & 293.01 & 0.001 & 0.34 & 1 & $\begin{array}{c}\text { Moderate } \\
\text { effect }\end{array}$ \\
\hline $\begin{array}{l}\text { Impact pressure on } \\
\text { ball }\left(\mathrm{KgF}^{-2}\right)\end{array}$ & $0.94 \pm 0.72^{\mathrm{a}, \mathrm{b}, \mathrm{c}}$ & $1.12 \pm 0.86^{\mathrm{a}}$ & $1.19 \pm 0.91^{b}$ & $1.13 \pm 0.88^{c}$ & 6.64 & 0.001 & 0.01 & 0.97 & No effect \\
\hline
\end{tabular}

Legend: amp: amplitude; $\mathrm{m}$ : meters; $\mathrm{m} . \mathrm{s}^{-1}$ : meters per second raise to the power of $-1 ; \mathrm{ms}$ : milliseconds; $\mathrm{m} . \mathrm{s}^{-2}$ : meters per second raise to the power of -1 ; m: meters; deg: degrees. KgF.cm ${ }^{-2}$ : impact pressure. * One-way ANOVA: significantly different at $p<0.05$ compared with (Scheffé or Games-Howell Post hoc). ( $\left.{ }^{a}\right)$ Practice Condition 1 vs. Practice Condition 2. ( ${ }^{b}$ ) Practice Condition 1 vs. Practice Condition 3. ${ }^{(c)}$ Practice Condition 1 vs. Practice Condition 4. $\left({ }^{\mathrm{d}}\right)$ Practice Condition 2 vs. Practice Condition 3. ( $\left.{ }^{\mathrm{e}}\right)$ Practice Condition 2 vs. Practice Condition 4. $\left({ }^{f}\right)$ Practice Condition 3 vs. Practice Condition 4.

Regarding the variables related to amplitude, it is possible to observe that both linear (m) and angular (deg) metrics tend to increase with the distance to the hole. In fact, statistically significant differences were found between all 4 PC for each of the amplitude variables: backswing/downswing amplitude $\left(\mathrm{F}(3,1677)=214.33 ; p\right.$-value $=0.001 ; \eta^{2}=0.28$; $\pi=1)$, the follow-through amplitude $\left(\mathrm{F}(3,1677)=332.12, p\right.$-value $\left.=0.001, \eta^{2}=0.37, \pi=1\right)$ and the maximum amplitude of the putting movement $(\mathrm{F}(3,1677)=511.72, p$-value $=0.001$, $\left.\eta^{2}=0.48, \pi=1\right)$.

In terms of inter-individual motor performance, the variation coefficient (see Table 3 in attachment) in the backswing/downswing phase ranged between 36\% (PC1) and 23\% (PC4). The results obtained for the follow-through show an increase in amplitude as the distance to the hole increases. The variation coefficient indicates an inter-individual variation between $23 \%$ and $30 \%$. For the maximum amplitude, the inter-individual variation values were relatively low, bellow $22 \%$ in all distances.

Additionally, maximum putting amplitude indicated that the majority of players made this movement with greater amplitude when the distance to the hole increased, as it may be confirmed in Table 4 . This was not the case for player 3, however, namely for PC3 and PC4.

Regarding the duration of each phase, the results of backswing duration (ms) indicated statistically significant differences between the beat distances $(F(3,1677)=10.51$, $p$-value $=0.001, \eta 2=0.02, \pi=0.99)$. We found longer backswings when comparing between PC1 and PC3 ( $p$-value $=0.006)$, PC1 and PC4 ( $p$-value $=0.001)$, and between PC2 and PC4 ( $p$-value $=0.006)$. On an inter-individual analysis of the backswing duration $(\mathrm{ms})$, 8 players $(1,5,7,8,11,12,13$ and 14) depicted a clear tendency to increase the duration time of this phase as the distance to the hole increased. In the remaining phases of putting, this tendency of proportionality is not so evident (Table 5 , in attachment). 
Table 3. Putting process and dependent variables in each distance to the hole.

\begin{tabular}{|c|c|c|c|c|c|}
\hline \multirow[b]{2}{*}{ Putting Process and Dependent Variables } & \multicolumn{5}{|c|}{ Practice Condition (PC) and Distance to the Hole (meters-m) } \\
\hline & Values & PC1 (1 m) & PC2 (2 m) & PC3 (3 m) & PC4 (4 m) \\
\hline \multirow{3}{*}{ Backswing/Downswing amplitude [deg] * } & M & 9.23 & 11.07 & 12.80 & 14.78 \\
\hline & SD & 2.72 & 2.91 & 3.17 & 4.27 \\
\hline & $\mathrm{VC} \%$ & 29 & 26 & 25 & 29 \\
\hline \multirow{3}{*}{ Backswing/Downswing amplitude * [m] } & $\mathrm{M}$ & 0.14 & 0.17 & 0.20 & 0.22 \\
\hline & SD & 0.05 & 0.05 & 0.05 & 0.05 \\
\hline & $\mathrm{VC} \%$ & 36 & 29 & 25 & 23 \\
\hline \multirow{3}{*}{ Follow-through amplitude [deg] } & $\mathrm{M}$ & 15.89 & 20.49 & 25.27 & 29.40 \\
\hline & SD & 4.5 & 6.08 & 6.84 & 8.32 \\
\hline & $\mathrm{VC} \%$ & 28 & 30 & 27 & 28 \\
\hline \multirow{3}{*}{ Follow-through amplitude [m] } & $\mathrm{M}$ & 0.29 & 0.35 & 0.43 & 0.48 \\
\hline & $\mathrm{SD}$ & 0.07 & 0.09 & 0.11 & 0.11 \\
\hline & $\mathrm{VC} \%$ & 25 & 27 & 26 & 23 \\
\hline \multirow{3}{*}{ Maximum putting amplitude [deg] } & $\mathrm{M}$ & 26.18 & 32.88 & 39.59 & 45.20 \\
\hline & SD & 5.59 & 6.97 & 8.11 & 8.79 \\
\hline & $\mathrm{VC} \%$ & 21 & 21 & 20 & 19 \\
\hline \multirow{3}{*}{ Maximum putting amplitude $[\mathrm{m}]$} & $\mathrm{M}$ & 0.461 & 0.567 & 0.678 & 0.749 \\
\hline & $\mathrm{SD}$ & 0.102 & 0.115 & 0.134 & 0.134 \\
\hline & $\mathrm{VC} \%$ & 22 & 20 & 20 & 18 \\
\hline \multirow{3}{*}{ Backswing duration [ms] } & $\mathrm{M}$ & 547.17 & 571.50 & 586.31 & 607.40 \\
\hline & SD & 172.73 & 157.19 & 143.73 & 161.53 \\
\hline & $\mathrm{VC} \%$ & 32 & 28 & 25 & 27 \\
\hline \multirow{3}{*}{ Downswing duration [ms] } & $\mathrm{M}$ & 298.21 & 309.90 & 300.36 & 297.12 \\
\hline & SD & 72.87 & 100.89 & 82.40 & 83.77 \\
\hline & $\mathrm{VC} \%$ & 24 & 33 & 27 & 28 \\
\hline \multirow{3}{*}{ Impact duration [ms] } & $\mathrm{M}$ & 0.70 & 0.70 & 0.65 & 0.65 \\
\hline & SD & 0.40 & 0.40 & 0.40 & 0.40 \\
\hline & $\mathrm{VC} \%$ & 57 & 57 & 62 & 62 \\
\hline \multirow{3}{*}{ Follow-through duration [ms] } & $\mathrm{M}$ & 399.76 & 400.90 & 421.45 & 412.19 \\
\hline & SD & 131.47 & 136.18 & 144.49 & 127.21 \\
\hline & $\mathrm{VC} \%$ & 33 & 34 & 34 & 31 \\
\hline \multirow{3}{*}{ Maximum duration [ms] } & $\mathrm{M}$ & 1259.31 & 1276.76 & 1308.64 & 1316.86 \\
\hline & SD & 297.29 & 302.83 & 297.78 & 295.18 \\
\hline & $\mathrm{VC} \%$ & 24 & 24 & 23 & 22 \\
\hline \multirow{3}{*}{ Impact velocity [deg.m $\left.{ }^{-1}\right]$} & $\mathrm{M}$ & 59.85 & 74.31 & 88.01 & 104.75 \\
\hline & SD & 8.07 & 8.34 & 10.61 & 19.30 \\
\hline & $\mathrm{VC} \%$ & 13 & 11 & 12 & 18 \\
\hline \multirow{3}{*}{ Speed of impact on the ball $\left[\mathrm{m} . \mathrm{s}^{-1}\right]$} & $\mathrm{M}$ & 1.11 & 1.39 & 1.69 & 1.90 \\
\hline & $\mathrm{SD}$ & 0.16 & 0.18 & 0.25 & 0.27 \\
\hline & $\mathrm{VC} \%$ & 14 & 13 & 15 & 14 \\
\hline \multirow{3}{*}{ Peak acceleration [deg.m ${ }^{-2}$ ] } & $\mathrm{M}$ & 1.11 & 1.39 & 1.69 & 1.90 \\
\hline & SD & 87.61 & 91.30 & 120.53 & 172.62 \\
\hline & $\mathrm{VC} \%$ & 31 & 27 & 30 & 34 \\
\hline \multirow{3}{*}{ Peak acceleration $\left[\mathrm{m} . \mathrm{s}^{-2}\right]$} & $\mathrm{M}$ & 4.67 & 5.76 & 6.94 & 7.89 \\
\hline & SD & 1.06 & 1.36 & 1.84 & 2.11 \\
\hline & $\mathrm{VC} \%$ & 23 & 24 & 27 & 27 \\
\hline \multirow{3}{*}{ Impact pressure on the ball $\left[\mathrm{KgF} . \mathrm{m}^{-2}\right]$} & $\mathrm{M}$ & 0.94 & 1.12 & 1.19 & 1.13 \\
\hline & SD & 0.72 & 0.86 & 0.91 & 0.88 \\
\hline & $\mathrm{VC} \%$ & 77 & 77 & 76 & 78 \\
\hline
\end{tabular}

Legend: $\mathrm{m}$ : meters; $\mathrm{m} . \mathrm{s}^{-1}:$ meters per second raise to the power of $-1 ; \mathrm{ms}$ : milliseconds; $\mathrm{m} . \mathrm{s}^{-2}$ : meters per second raise to the power of $-1 ; \mathrm{m}$ : meters; deg: degrees. KgF. $\mathrm{cm}^{-2}$ : impact pressure instead. PC: Practice Condition (PC1: Practice Condition 1; PC2: Practice Condition 2; PC3: Practice Condition 3; PC4: Practice Condition 4). Overall results that encompass all golf players that performed 30 trials each. * Amplitudes of both backswing and the downswing are the same. The backswing starts with the putter near the ball, thus moving far away from it. The farthest point corresponds to the transition between the backswing (ending) and the downswing (starting). As expected, the downswing ends when the putter moves near the ball once again $[2,8]$. 
Table 4. Backswing/Downswing amplitude [deg and $\mathrm{m}$ ], Follow-through amplitude [deg and m] and Maximum amplitude [deg and $\mathrm{m}]$.

\begin{tabular}{|c|c|c|c|c|c|c|c|c|}
\hline \multirow[b]{3}{*}{ Players } & \multicolumn{8}{|c|}{ Distance to the hole: } \\
\hline & \multicolumn{4}{|c|}{ Backswing/Downswing Amplitude [deg] } & \multicolumn{4}{|c|}{ Backswing/Downswing Amplitude [m] } \\
\hline & $1 \mathrm{~m}$ & $2 \mathrm{~m}$ & $3 \mathrm{~m}$ & $4 \mathrm{~m}$ & $1 \mathrm{~m}$ & $2 \mathrm{~m}$ & $3 \mathrm{~m}$ & $4 \mathrm{~m}$ \\
\hline 1 & $13.77 \pm 0.95$ & $16.22 \pm 2.80$ & $15.69 \pm 1.10$ & $25.39 \pm 2.95$ & $0.17 \pm 0.01$ & $0.22 \pm 0.05$ & $0.24 \pm 0,02$ & $0.25 \pm 0.05$ \\
\hline 2 & $11.55 \pm 0.79$ & $13.28 \pm 0.73$ & $17.13 \pm 1.63$ & $18.63 \pm 1.62$ & $0.19 \pm 0.01$ & $0.23 \pm 0.01$ & $0.26 \pm 0.02$ & $0.28 \pm 0.02$ \\
\hline 3 & $11.91 \pm 1.40$ & $13.07 \pm 1.26$ & $15.67 \pm 2.20$ & $16.16 \pm 1.92$ & $0.18 \pm 0.02$ & $0.20 \pm 0.02$ & $0.23 \pm 0.03$ & $0.21 \pm 0.02$ \\
\hline 4 & $4.73 \pm 0.46$ & $6.50 \pm 0.53$ & $7.91 \pm 0.42$ & $8.22 \pm 0.74$ & $0.07 \pm 0.01$ & $0.09 \pm 0.01$ & $0.11 \pm 0.01$ & $0.11 \pm 0.01$ \\
\hline 5 & $5.90 \pm 1.01$ & $7.63 \pm 1.29$ & $8.97 \pm 0.60$ & $10.32 \pm 0.67$ & $0.09 \pm 0.01$ & $0.12 \pm 0.02$ & $0.14 \pm 0.01$ & $0.16 \pm 0.01$ \\
\hline 6 & $9.62 \pm 2.21$ & $9.18 \pm 0.55$ & $10.10 \pm 0.71$ & $11.57 \pm 1.38$ & $0.13 \pm 0.04$ & $0.15 \pm 0.03$ & $0.16 \pm 0.01$ & $0.18 \pm 0.02$ \\
\hline 7 & $8.13 \pm 0.75$ & $10.21 \pm 0.73$ & $11.35 \pm 1.83$ & $13.74 \pm 0.84$ & $0.15 \pm 0.01$ & $0.19 \pm 0.01$ & $0.20 \pm 0.03$ & $0.24 \pm 0.02$ \\
\hline 8 & $8.08 \pm 0.58$ & $9.54 \pm 0.65$ & $11.39 \pm 1.15$ & $12.68 \pm 1.40$ & $0.13 \pm 0.01$ & $0.16 \pm 0.01$ & $0.19 \pm 0.02$ & $0.21 \pm 0.01$ \\
\hline 9 & $7.60 \pm 0.78$ & $10.56 \pm 3.28$ & $10.58 \pm 0.85$ & $12.90 \pm 0.68$ & $0.14 \pm 0.07$ & $0.18 \pm 0.07$ & $0.18 \pm 0.01$ & $0.23 \pm 0.01$ \\
\hline 10 & $8.94 \pm 0.85$ & $12.01 \pm 0.77$ & $14.58 \pm 1.20$ & $16.12 \pm 0.82$ & $0.14 \pm 0.01$ & $0.18 \pm 0.01$ & $0.21 \pm 0.02$ & $0.23 \pm 0.01$ \\
\hline 11 & $10.42 \pm 1.78$ & $13.54 \pm 1.70$ & $15.87 \pm 1.74$ & $15.97 \pm 1.38$ & $0.15 \pm 0.02$ & $0.20 \pm 0.02$ & $0.24 \pm 0.03$ & $0.25 \pm 0.02$ \\
\hline 12 & $10.54 \pm 0.57$ & $12.03 \pm 1.17$ & $14.91 \pm 0.70$ & $15.81 \pm 1.54$ & $0.17 \pm 0.01$ & $0.20 \pm 0.02$ & $0.25 \pm 0.03$ & $0.25 \pm 0.02$ \\
\hline 13 & $6.62 \pm 0.54$ & $8.97 \pm 0.65$ & $10.29 \pm 1.03$ & $12.44 \pm 0.99$ & $0.11 \pm 0.01$ & $0.14 \pm 0.01$ & $0.17 \pm 0.02$ & $0.21 \pm 0.04$ \\
\hline \multirow[t]{3}{*}{14} & $11.33 \pm 1.95$ & $12.30 \pm 0.82$ & $14.81 \pm 1.10$ & $16.94 \pm 1.50$ & $0.19 \pm 0.04$ & $0.21 \pm 0.01$ & $0.25 \pm 0.02$ & $0.28 \pm 0.02$ \\
\hline & \multicolumn{8}{|c|}{ Distance to the hole: } \\
\hline & \multicolumn{4}{|c|}{ Follow-through amplitude [deg] } & \multicolumn{4}{|c|}{ Follow-through amplitude [m] } \\
\hline Players & $1 \mathrm{~m}$ & $2 \mathrm{~m}$ & $3 \mathrm{~m}$ & $4 \mathrm{~m}$ & $1 \mathrm{~m}$ & $2 \mathrm{~m}$ & $3 \mathrm{~m}$ & $4 \mathrm{~m}$ \\
\hline 1 & $20.29 \pm 2.25$ & $27.45 \pm 4.56$ & $29.08 \pm 2.31$ & $30.34 \pm 10.35$ & $0.28 \pm 0.05$ & $0.40 \pm 0.08$ & $0.45 \pm 0.09$ & $0.45 \pm 0.14$ \\
\hline 2 & $14.97 \pm 2.16$ & $21.80 \pm 3.09$ & $28.83 \pm 3.39$ & $34.90 \pm 2.78$ & $0.31 \pm 0.05$ & $0.43 \pm 0.06$ & $0.56 \pm 0.06$ & $0.60 \pm 0.06$ \\
\hline 3 & $14.25 \pm 2.38$ & $22.95 \pm 3.92$ & $30.39 \pm 6.88$ & $40.86 \pm 6.33$ & $0.28 \pm 0.05$ & $0.40 \pm 0.14$ & $0.52 \pm 0.12$ & $0.54 \pm 0.08$ \\
\hline 4 & $21.47 \pm 1.45$ & $31.03 \pm 2.09$ & $36.29 \pm 2.66$ & $41.42 \pm 4.13$ & $0.35 \pm 0.06$ & $0.48 \pm 0.07$ & $0.61 \pm 0.08$ & $0.62 \pm 0.08$ \\
\hline 5 & $14.66 \pm 2.03$ & $18.93 \pm 3.52$ & $24.11 \pm 3.67$ & $29.01 \pm 3.39$ & $0.28 \pm 0.03$ & $0.35 \pm 0.06$ & $0.44 \pm 0.05$ & $0.52 \pm 0.04$ \\
\hline 6 & $15.03 \pm 5.41$ & $14.36 \pm 1.48$ & $18.38 \pm 5.36$ & $17.60 \pm 3.12$ & $0.24 \pm 0.07$ & $0.27 \pm 0.05$ & $0.34 \pm 0.10$ & $0.33 \pm 0.06$ \\
\hline 7 & $12.85 \pm 1.43$ & $15.04 \pm 1.19$ & $19.71 \pm 3.89$ & $24.41 \pm 2.60$ & $0.28 \pm 0.03$ & $0.31 \pm 0.03$ & $0.39 \pm 0.03$ & $0.46 \pm 0.04$ \\
\hline 8 & $23.00 \pm 1.27$ & $24.00 \pm 1.55$ & $27.71 \pm 4.88$ & $31.03 \pm 2.70$ & $0.37 \pm 0.03$ & $0.37 \pm 0.04$ & $0.41 \pm 0.06$ & $0.45 \pm 0.06$ \\
\hline 9 & $13.64 \pm 2.37$ & $19.36 \pm 5.02$ & $26.35 \pm 1.95$ & $30.84 \pm 2.35$ & $0.23 \pm 0.06$ & $0.33 \pm 0.07$ & $0.45 \pm 0.04$ & $0.54 \pm 0.05$ \\
\hline 10 & $19.41 \pm 3.14$ & $24.49 \pm 2.60$ & $27.42 \pm 2.43$ & $30.13 \pm 3.11$ & $0.34 \pm 0.07$ & $0.36 \pm 0.04$ & $0.38 \pm 0.03$ & $0.41 \pm 0.04$ \\
\hline 11 & $17.57 \pm 2.63$ & $24.15 \pm 2.76$ & $31.13 \pm 4.75$ & $35.93 \pm 3.85$ & $0.26 \pm 0.07$ & $0.33 \pm 0.07$ & $0.45 \pm 0.07$ & $0.56 \pm 0.07$ \\
\hline 12 & $13.37 \pm 1.87$ & $15.62 \pm 3.06$ & $18.69 \pm 2.23$ & $21.95 \pm 3.46$ & $0.31 \pm 0.04$ & $0.39 \pm 0.08$ & $0.43 \pm 0.08$ & $0.47 \pm 0.08$ \\
\hline 13 & $9.60 \pm 0.82$ & $12.38 \pm 0.93$ & $16.46 \pm 1.43$ & $19.15 \pm 1.85$ & $0.18 \pm 0.03$ & $0.20 \pm 0.02$ & $0.26 \pm 0.02$ & $0.31 \pm 0.08$ \\
\hline \multirow[t]{3}{*}{14} & $12.30 \pm 2.67$ & $15.26 \pm 1.41$ & $19.17 \pm 1.90$ & $24.02 \pm 2.12$ & $0.29 \pm 0.05$ & $0.32 \pm 0.03$ & $0.40 \pm 0.03$ & $0.48 \pm 0.04$ \\
\hline & \multicolumn{8}{|c|}{ Distance to the hole: } \\
\hline & \multicolumn{4}{|c|}{ Maximum amplitude [deg] } & \multicolumn{4}{|c|}{ Maximum amplitude [m] } \\
\hline Players & $1 \mathrm{~m}$ & $2 \mathrm{~m}$ & $3 \mathrm{~m}$ & $4 \mathrm{~m}$ & $1 \mathrm{~m}$ & $2 \mathrm{~m}$ & $3 \mathrm{~m}$ & $4 \mathrm{~m}$ \\
\hline 1 & $36.03 \pm 2.64$ & $45.69 \pm 4.11$ & $47.20 \pm 2.21$ & $51.20 \pm 5.00$ & $0.48 \pm 0.07$ & $0.67 \pm 0.10$ & $0.72 \pm 0.11$ & $0.76 \pm 0.14$ \\
\hline 2 & $27.26 \pm 2.28$ & $35.79 \pm 2.81$ & $47.07 \pm 3.86$ & $54.85 \pm 2.33$ & $0.56 \pm 0.06$ & $0.72 \pm 0.07$ & $0.88 \pm 0.06$ & $0.95 \pm 0.07$ \\
\hline 3 & $26.88 \pm 3.17$ & $36.88 \pm 3.99$ & $47.77 \pm 5.61$ & $58.32 \pm 6.72$ & $0.50 \pm 0.06$ & $0.64 \pm 0.15$ & $0.80 \pm 0.11$ & $0.79 \pm 0.09$ \\
\hline 4 & $27.39 \pm 1.45$ & $39.08 \pm 2.17$ & $46.01 \pm 2.72$ & $51.73 \pm 3.96$ & $0.45 \pm 0.06$ & $0.61 \pm 0.08$ & $0.78 \pm 0.09$ & $0.79 \pm 0.09$ \\
\hline 5 & $21.40 \pm 3.24$ & $27.67 \pm 2.98$ & $33.91 \pm 3.77$ & $40.26 \pm 3.54$ & $0.40 \pm 0.03$ & $0.52 \pm 0.05$ & $0.64 \pm 0.05$ & $0.73 \pm 0.05$ \\
\hline 6 & $25.78 \pm 6.35$ & $25.15 \pm 1.38$ & $30.15 \pm 5.29$ & $31.13 \pm 2.28$ & $0.39 \pm 0.13$ & $0.47 \pm 0.08$ & $0.52 \pm 0.09$ & $0.53 \pm 0.05$ \\
\hline 7 & $22.34 \pm 1.28$ & $26.67 \pm 1.52$ & $32.44 \pm 5.83$ & $39.75 \pm 2.73$ & $0.46 \pm 0.03$ & $0.53 \pm 0.03$ & $0.63 \pm 0.05$ & $0.74 \pm 0.05$ \\
\hline 8 & $32.64 \pm 1.62$ & $35.36 \pm 1.68$ & $41.36 \pm 6.15$ & $46.10 \pm 2.69$ & $0.51 \pm 0.04$ & $0.53 \pm 0.04$ & $0.60 \pm 0.09$ & $0.67 \pm 0.07$ \\
\hline 9 & $21.82 \pm 2.61$ & $30.94 \pm 2.02$ & $37.16 \pm 1.82$ & $44.20 \pm 2.16$ & $0.39 \pm 0.14$ & $0.55 \pm 0.10$ & $0.66 \pm 0.04$ & $0.81 \pm 0.05$ \\
\hline 10 & $29.65 \pm 2.94$ & $38.42 \pm 2.51$ & $44.46 \pm 2.75$ & $49.13 \pm 3.11$ & $0.52 \pm 0.07$ & $0.58 \pm 0.04$ & $0.64 \pm 0.04$ & $0.69 \pm 0.05$ \\
\hline 11 & $29.25 \pm 2.93$ & $39.62 \pm 3.62$ & $49.50 \pm 4.36$ & $53.98 \pm 4.31$ & $0.43 \pm 0.09$ & $0.55 \pm 0.08$ & $0.74 \pm 0.08$ & $0.88 \pm 0.09$ \\
\hline 12 & $24.39 \pm 1.97$ & $28.07 \pm 2.30$ & $34.05 \pm 2.38$ & $38.06 \pm 2.72$ & $0.52 \pm 0.05$ & $0.64 \pm 0.07$ & $0.73 \pm 0,12$ & $0.76 \pm 0,11$ \\
\hline 13 & $17.28 \pm 0.99$ & $22.78 \pm 1.24$ & $28.56 \pm 2.01$ & $33.18 \pm 2.18$ & $0.31 \pm 0.02$ & $0.36 \pm 0.02$ & $0.45 \pm 0.03$ & $0.56 \pm 0.07$ \\
\hline 14 & $24.40 \pm 4.67$ & $28.23 \pm 1.26$ & $34.61 \pm 2.05$ & $40.88 \pm 2.47$ & $0.52 \pm 0.10$ & $0.59 \pm 0.03$ & $0.71 \pm 0.04$ & $0.82 \pm 0.06$ \\
\hline
\end{tabular}


Table 5. Backswing duration [ms], Downswing duration [ms], Impact duration [ms], Follow-through duration [ms] and Maximum duration [ms].

\begin{tabular}{|c|c|c|c|c|c|c|c|c|}
\hline \multirow[b]{3}{*}{ Players } & \multicolumn{8}{|c|}{ Distance to the hole: } \\
\hline & \multirow{2}{*}{\multicolumn{3}{|c|}{$\begin{array}{l}\text { Backswing Duration [ms] } \\
2 \mathrm{~m} \\
3 \mathrm{~m}\end{array}$}} & \multirow{2}{*}{\multicolumn{2}{|c|}{$4 \mathrm{~m} \quad 1 \mathrm{~m}$}} & \multicolumn{2}{|c|}{ Downswing Duration [ms] } & \multirow[b]{2}{*}{$4 \mathrm{~m}$} \\
\hline & & & & & & $2 \mathrm{~m}$ & $3 \mathrm{~m}$ & \\
\hline 1 & $809.67 \pm 237.70$ & $835 \pm 204.12$ & $863.33 \pm 191.93$ & $984 \pm 254.45$ & $430.67 \pm 19.82$ & $469 \pm 129.21$ & $444.00 \pm 24.17$ & $449.67 \pm 24.96$ \\
\hline 2 & $716.67 \pm 67.89$ & $720.33 \pm 40.21$ & $772.00 \pm 59.07$ & $754.33 \pm 44.25$ & $419.00 \pm 33.30$ & $381.33 \pm 25.79$ & $374.67 \pm 21.09$ & $350.33 \pm 27.26$ \\
\hline 3 & $479.33 \pm 33.36$ & $516.33 \pm 38.86$ & $521.67 \pm 44.20$ & $510 \pm 31.73$ & $288.00 \pm 20.72$ & $259.67 \pm 19.23$ & $263.67 \pm 37.64$ & $241.33 \pm 21.87$ \\
\hline 4 & $554.33 \pm 48.08$ & $595.67 \pm 39.22$ & $573.00 \pm 40.59$ & $592.67 \pm 41.87$ & $186.33 \pm 32.71$ & $143.33 \pm 26.37$ & $130.33 \pm 12.24$ & $111.67 \pm 16.75$ \\
\hline 5 & $357.33 \pm 21.44$ & $364.33 \pm 19.78$ & $387.67 \pm 13.34$ & $393.67 \pm 17.79$ & $210.33 \pm 15.81$ & $211.33 \pm 32.84$ & $211.33 \pm 12.04$ & $209.00 \pm 8.31$ \\
\hline 6 & $722.67 \pm 217.09$ & $759 \pm 194.74$ & $691.67 \pm 37.16$ & $728.67 \pm 54.94$ & $307.67 \pm 33.93$ & $308.67 \pm 13.35$ & $292.67 \pm 16.11$ & $291.00 \pm 15.99$ \\
\hline 7 & $462.00 \pm 24.82$ & $495.67 \pm 25.91$ & $527.67 \pm 99.76$ & $534.33 \pm 100.62$ & $263.33 \pm 19.38$ & $270.67 \pm 12.89$ & $259.33 \pm 11.53$ & $267.00 \pm 12.69$ \\
\hline 8 & $529.00 \pm 42.06$ & $601.67 \pm 81.82$ & $665.67 \pm 101.90$ & $667.33 \pm 61.37$ & $284.67 \pm 33.24$ & $306.33 \pm 28.22$ & $342.33 \pm 39.72$ & $333.33 \pm 17.00$ \\
\hline 9 & $600.33 \pm 283.52$ & $591 \pm 199.35$ & $553.67 \pm 131.14$ & $556.33 \pm 69.88$ & $231.00 \pm 21.35$ & $279.67 \pm 167.20$ & $238.00 \pm 17.20$ & $276.33 \pm 25.88$ \\
\hline 10 & $535.67 \pm 55.06$ & $499 \pm 33.30$ & $522.00 \pm 33.61$ & $535 \pm 22.91$ & $338.33 \pm 48.17$ & $361.00 \pm 37.36$ & $371.00 \pm 29.14$ & $400.00 \pm 19.49$ \\
\hline 11 & $492.00 \pm 92.28$ & $546.67 \pm 39.44$ & $592.67 \pm 42.26$ & $637.67 \pm 54.81$ & $346.00 \pm 41.28$ & $391.67 \pm 37.95$ & $399.00 \pm 31.24$ & $378.00 \pm 31.77$ \\
\hline 12 & $464.33 \pm 34.22$ & $510.67 \pm 40.41$ & $542.67 \pm 26.20$ & $573 \pm 40.59$ & $283.00 \pm 14.64$ & $273.33 \pm 34.48$ & $275.00 \pm 10.25$ & $269.00 \pm 21.35$ \\
\hline 13 & $502.67 \pm 65.62$ & $516.67 \pm 22.11$ & $527.67 \pm 27.41$ & $567.33 \pm 113.37$ & $281.33 \pm 21.09$ & $301.33 \pm 13.60$ & $300.00 \pm 23.80$ & $291.00 \pm 21.81$ \\
\hline \multirow[t]{3}{*}{14} & $434.33 \pm 22.46$ & $449 \pm 25.08$ & $467.00 \pm 21.16$ & $469.33 \pm 18.43$ & $305.33 \pm 13.84$ & $303.67 \pm 14.94$ & $311.00 \pm 15.35$ & $294 \pm 12.54$ \\
\hline & \multicolumn{8}{|c|}{ Distance to the hole: } \\
\hline & \multicolumn{4}{|c|}{ Impact duration [ms] } & \multicolumn{4}{|c|}{ Follow-through duration [ms] } \\
\hline Players & $1 \mathrm{~m}$ & $2 \mathrm{~m}$ & $3 \mathrm{~m}$ & $4 \mathrm{~m}$ & $1 \mathrm{~m}$ & $2 \mathrm{~m}$ & $3 \mathrm{~m}$ & $4 \mathrm{~m}$ \\
\hline 1 & $0.94 \pm 0.43$ & $0.88 \pm 0.53$ & $0.66 \pm 0.36$ & $0.92 \pm 0.50$ & $515.67 \pm 120.24$ & $672.00 \pm 156.62$ & $690.67 \pm 109.27$ & $602.00 \pm 140.22$ \\
\hline 2 & $0.96 \pm 0.32$ & $0.90 \pm 0.38$ & $0.59 \pm 0.48$ & $0.57 \pm 0.28$ & $410.00 \pm 71.18$ & $469.33 \pm 95.84$ & $528.67 \pm 54.45$ & $521.67 \pm 51.58$ \\
\hline 3 & $0.52 \pm 0.38$ & $0.66 \pm 0.53$ & $0.72 \pm 0.52$ & $0.72 \pm 0.36$ & $325.67 \pm 71.68$ & $424.67 \pm 139.68$ & $468.33 \pm 160.38$ & $530.33 \pm 126.61$ \\
\hline 4 & $0.92 \pm 0.45$ & $0.81 \pm 0.39$ & $0.80 \pm 0.29$ & $0.84 \pm 0.49$ & $528.67 \pm 86.25$ & $530.67 \pm 52.40$ & $528.33 \pm 57.91$ & $512.00 \pm 60.13$ \\
\hline 5 & $0.75 \pm 0.43$ & $0.56 \pm 0.44$ & $0.47 \pm 0.44$ & $0.41 \pm 0.42$ & $306.00 \pm 18.55$ & $302.33 \pm 34.22$ & $312.00 \pm 21.35$ & $309.33 \pm 21.44$ \\
\hline 6 & $0.52 \pm 0.41$ & $0.61 \pm 0.35$ & $0.67 \pm 0.40$ & $0.56 \pm 0.29$ & $591.33 \pm 204.97$ & $313.33 \pm 86.38$ & $376.00 \pm 151.69$ & $325.00 \pm 80.11$ \\
\hline 7 & $0.71 \pm 0.22$ & $0.84 \pm 0.36$ & $0.62 \pm 0.47$ & $0.64 \pm 0.38$ & $299.33 \pm 24.35$ & $303.67 \pm 10.48$ & $332.00 \pm 119.15$ & $314.33 \pm 12.30$ \\
\hline 8 & $0.83 \pm 0.37$ & $0.79 \pm 0.23$ & $0.64 \pm 0.24$ & $0.63 \pm 0.26$ & $535.33 \pm 51.88$ & $536.33 \pm 31.25$ & $557.33 \pm 69.28$ & $546.67 \pm 44.07$ \\
\hline 9 & $0.59 \pm 0.34$ & $0.44 \pm 0.27$ & $0.46 \pm 0.39$ & $0.43 \pm 0.48$ & $354.67 \pm 29.97$ & $407.67 \pm 81.15$ & $386.33 \pm 20.08$ & $392.67 \pm 18.79$ \\
\hline 10 & $0.77 \pm 0.39$ & $0.80 \pm 0.26$ & $0.68 \pm 0.25$ & $0.70 \pm 0.35$ & $382.33 \pm 65.86$ & $341.00 \pm 28.91$ & $328.00 \pm 18.87$ & $332.00 \pm 17.40$ \\
\hline 11 & $0.62 \pm 0.32$ & $0.69 \pm 0.38$ & $0.82 \pm 0.43$ & $0.65 \pm 0.40$ & $396.33 \pm 129.01$ & $392.67 \pm 58.42$ & $444.33 \pm 125.10$ & $451.00 \pm 111.55$ \\
\hline 12 & $0.77 \pm 0.17$ & $0.75 \pm 0.22$ & $0.70 \pm 0.23$ & $0.71 \pm 0.35$ & $317.67 \pm 25.78$ & $333.00 \pm 81.29$ & $341.00 \pm 59.24$ & $329.00 \pm 52.05$ \\
\hline 13 & $0.40 \pm 0.33$ & $0.57 \pm 0.46$ & $0.62 \pm 0.33$ & $0.62 \pm 0.43$ & $267.67 \pm 18.92$ & $256.00 \pm 14.74$ & $277.33 \pm 20.81$ & $274.00 \pm 11.14$ \\
\hline \multirow[t]{2}{*}{14} & $0.46 \pm 0.30$ & $0.56 \pm 0.33$ & $0.70 \pm 0.45$ & $0.64 \pm 0.23$ & $366.00 \pm 59.08$ & $330.00 \pm 23.66$ & $330.00 \pm 20.66$ & $330.67 \pm 19.48$ \\
\hline & \multicolumn{8}{|c|}{$\begin{array}{l}\text { Distance to the hole: } \\
\text { Maximum duration [ms] }\end{array}$} \\
\hline Players & \multicolumn{2}{|c|}{$1 \mathrm{~m}$} & \multicolumn{2}{|c|}{$2 \mathrm{~m}$} & \multicolumn{2}{|c|}{$3 \mathrm{~m}$} & \multicolumn{2}{|c|}{$4 \mathrm{~m}$} \\
\hline 1 & \multicolumn{2}{|c|}{$1756.00 \pm 262.41$} & \multicolumn{2}{|c|}{$1976.00 \pm 344.52$} & \multicolumn{2}{|c|}{$1998.00 \pm 228.29$} & \multicolumn{2}{|c|}{$2035.67 \pm 300.01$} \\
\hline 2 & 1545.67 & 131.85 & 1571.0 & 106.59 & 1675.33 & 98.99 & 1626.33 & \pm 87.92 \\
\hline 3 & 1093.0 & 89.75 & 1200.6 & 163.40 & 1253.67 & 159.53 & 1281.67 & $=139.21$ \\
\hline 4 & 1269.3 & 95.71 & 1269.6 & 75.30 & 1231.67 & 51.52 & 1216.33 & \pm 73.28 \\
\hline 5 & 873.67 & 33.61 & 878.0 & 34.58 & 911.00 & 28.09 & 912.00 & $=32.19$ \\
\hline 6 & 1621.67 & 351.36 & 1381.0 & 201.14 & 1360.33 & 155.98 & 1344.67 & $=107.85$ \\
\hline 7 & 1024.6 & 39.05 & 1070.0 & 29.55 & 1119.00 & 176.94 & 1115.67 & $=106.92$ \\
\hline 8 & 1349.0 & 80.01 & 1444.3 & 95.84 & 1565.33 & 151.23 & 1547.33 & \pm 73.71 \\
\hline 9 & 1186.00 & 259.58 & 1278.3 & 334.60 & 1178.00 & 132.83 & 1225.33 & \pm 78.52 \\
\hline 10 & 1256.3 & 83.96 & 1201.0 & 46.29 & 1221.00 & 47.07 & 1267.00 & \pm 33.88 \\
\hline 11 & 1234.33 & 163.76 & 1331.0 & 79.97 & 1436.00 & 118.73 & 1466.67 & $=124.86$ \\
\hline 12 & 1065.0 & 43.72 & 1117.0 & 92.71 & 1158.67 & 71.54 & 1171.00 & \pm 77.69 \\
\hline 13 & 1051.6 & 76.73 & 1074.0 & 33.43 & 1105.00 & 50.45 & 1132.33 & $=119.71$ \\
\hline 14 & 1105.6 & 73.24 & 1082.6 & 38.38 & 1108.00 & $=32.80$ & 1094.00 & \pm 27.40 \\
\hline
\end{tabular}

In fact, despite a significant number of players presented slightly higher values in the downswing duration (ms) in PC1 compared to the remaining practice conditions (see Table 5 , particularly players $2,3,4,6,8,10,12$ ), there were no statistically significant differences between the four PC for this putting phase $(\mathrm{F}(3,1677)=1.95 ; p$-value $=0.120$; $\eta^{2}=0.003 ; \pi=0.505$ ). Similarly, the follow-through duration (ms) remained steady as the distance to the hole increased, with no statistically significant differences between the four $\mathrm{PC}\left(\mathrm{F}(3,1677)=1.948 ; p\right.$-value $\left.=0.120 ; \eta^{2}=0.003 ; \pi=0.505\right)$. Regarding maximum duration $(\mathrm{ms})$, There were differences between PC1 and PC4 $(\mathrm{F}(3,1677)=3.44, p$-value $=0.02$, $\left.\eta^{2}=0.01, \pi=0.77\right)$, with a very low effect size value.

All velocity and acceleration-related variables showed significant differences for all PC. Regarding impact velocity (deg.sec ${ }^{-1}$ ), results indicate that most players showed an increase in motor performance values as the distance to the hole increased with statistically significant differences between all four PC $(\mathrm{F}(3,1677)=997.23 ; p$-value $=0.001 ; \eta 2=0.64$; $\pi=1)$. The impact velocity increased significantly with increasing distance to the hole, namely PC1 for PC2 ( $p$-value $=0.001)$, PC2 for PC3 $(p$-value $=0.001)$ and PC3 for PC4 
$(p$-value $=0.001)$. Low variation coefficients were also observed, and the impact velocity (deg) was stable throughout the four PC (see Table 3, in attachment).

Most players had higher peak acceleration $\left(\mathrm{deg} \cdot \mathrm{m}^{-2}\right)$ values, in line with the increase in distance to the hole (Table 6, in attachment). This was not the case of player 9, from PC3 to PC4 (see Table 5 , in attachment). There were statistically significant differences between all four PC $\left(\mathrm{F}(3,1677)=256.07 ; p\right.$-value $\left.=0.001 ; \eta^{2}=0.31 ; \pi=1\right)$. Peak acceleration $\left(\mathrm{deg} \cdot \mathrm{m}^{-2}\right)$ increased significantly with increasing distance to the hole, in PC1 to PC2 $(p$-value $=0.001)$, PC2 to PC3 ( $p$-value $=0.001)$, and PC3 for PC4 $(p$-value $=0.001)$. Regarding Peak velocity $\left(\right.$ deg. $\left.\mathrm{m}^{-1}\right)$, results show that an increase in the distance to the hole allowed a progressive increase of the values obtained in this putting phase. Compared to maximum amplitude (deg), impact velocity and peak velocity $\left(\right.$ deg. $\mathrm{m}^{-1}$ ) values varied substantially, with $\mathrm{CV}$ values ranging between $27 \%$ and $34 \%$.

Table 6. Impact Velocity [deg.sec ${ }^{-1}$, Speed of impact on the ball $\left[\mathrm{m} . \mathrm{s}^{-1}\right]$, Peak acceleration $\left[\mathrm{deg} \cdot \mathrm{sec}^{-1} \mathrm{~m} . \mathrm{s}^{-2}\right]$, and Impact pressure on the ball $\left[\mathrm{KgF} . \mathrm{m}^{-2}\right]$.

\begin{tabular}{|c|c|c|c|c|c|c|c|c|}
\hline \multirow[b]{3}{*}{ Players } & \multicolumn{8}{|c|}{ Distance to the hole: } \\
\hline & \multirow{2}{*}{\multicolumn{3}{|c|}{$\begin{array}{l}\text { Impact Velocity [deg. } \mathrm{sec}^{-1} \text { ] } \\
2 \mathrm{~m} \\
3 \mathrm{~m}\end{array}$}} & & \multicolumn{4}{|c|}{ Speed of Impact on the Ball $\left[\mathrm{m} . \mathrm{s}^{-1}\right]$} \\
\hline & & & & $4 \mathrm{~m}$ & $1 \mathrm{~m}$ & $2 \mathrm{~m}$ & $3 \mathrm{~m}$ & $4 \mathrm{~m}$ \\
\hline 1 & $70.23 \pm 4.78$ & $73.89 \pm 6.75$ & $81.15 \pm 3.68$ & $154.68 \pm 21.53$ & $0.97 \pm 0.09$ & $1.22 \pm 0.12$ & $1.43 \pm 0.18$ & $1.51 \pm 0.29$ \\
\hline 2 & $57.76+2.99$ & $79.36+3.73$ & $93.08+6.13$ & $110.78+7.47$ & $1.14+0.09$ & $1.54+0.11$ & $1.95+0.10$ & $2.17+0.10$ \\
\hline 3 & $67.68 \pm 4.30$ & $88.67 \pm 4.13$ & $106.89 \pm 5.73$ & $119.71 \pm 9.54$ & $1.18 \pm 0.08$ & $1.44 \pm 0.17$ & $1.79 \pm 0.10$ & $1.77 \pm 0.11$ \\
\hline 4 & $63.53 \pm 3.35$ & $85.45 \pm 6.06$ & $105.28 \pm 4.54$ & $119.37 \pm 8.84$ & $1.24 \pm 0.11$ & $1.65 \pm 0.16$ & $2.09 \pm 0.14$ & $2.36 \pm 0.14$ \\
\hline 5 & $56.33 \pm 7.89$ & $71.43 \pm 5.38$ & $85.26 \pm 3.54$ & $99.51 \pm 4.64$ & $1.18 \pm 0.09$ & $1.57 \pm 0.15$ & $1.93 \pm 0.13$ & $2.23 \pm 0.13$ \\
\hline 6 & $59.74 \pm 3.69$ & $71.83 \pm 5.17$ & $83.35 \pm 6.19$ & $97.50 \pm 6.64$ & $0.93 \pm 0.16$ & $1.30 \pm 0.16$ & $1.56 \pm 0.14$ & $1.66 \pm 0.12$ \\
\hline 7 & $58.37 \pm 3.28$ & $69.37 \pm 3.59$ & $81.86 \pm 14.08$ & $96.43 \pm 4.46$ & $1.21 \pm 0.08$ & $1.37 \pm 0.08$ & $1.67 \pm 0.25$ & $1.99 \pm 0.10$ \\
\hline 8 & $67.03 \pm 2.25$ & $74.24 \pm 2.63$ & $83.21 \pm 11.86$ & $94.55 \pm 8.63$ & $1.27 \pm 0.07$ & $1.32 \pm 0.08$ & $1.54 \pm 0.23$ & $1.74 \pm 0.17$ \\
\hline 9 & $52.59 \pm 7.88$ & $69.17 \pm 3.98$ & $75.65 \pm 3.44$ & $84.64 \pm 3.17$ & $1.00 \pm 0.11$ & $1.34 \pm 0.10$ & $1.61 \pm 0.07$ & $1.86 \pm 0.11$ \\
\hline 10 & $58.96 \pm 3.55$ & $78.87 \pm 2.84$ & $93.51 \pm 4.26$ & $99.70 \pm 4.30$ & $1.16 \pm 0.12$ & $1.43 \pm 0.08$ & $1.64 \pm 0.07$ & $1.77 \pm 0.09$ \\
\hline 11 & $60.11 \pm 5.84$ & $75.78 \pm 6.00$ & $90.70 \pm 7.10$ & $103.72 \pm 4.95$ & $0.94 \pm 0.15$ & $1.15 \pm 0.12$ & $1.49 \pm 0.13$ & $1.88 \pm 0.11$ \\
\hline 12 & $59.95 \pm 3.85$ & $71.32 \pm 8.63$ & $89.07 \pm 3.95$ & $98.43 \pm 7.80$ & $1.23 \pm 0.10$ & $1.53 \pm 0.11$ & $1.82 \pm 0.24$ & $1.95 \pm 0.23$ \\
\hline 13 & $53.78 \pm 3.23$ & $67.53 \pm 4.61$ & $84.57 \pm 3.89$ & $103.68 \pm 6.28$ & $1.02 \pm 0.09$ & $1.26 \pm 0.07$ & $1.50 \pm 0.10$ & $1.83 \pm 0.21$ \\
\hline \multirow[t]{3}{*}{14} & $55.16 \pm 9.91$ & $63.48 \pm 3.29$ & $76.20 \pm 4.06$ & $83.83 \pm 8.33$ & $1.13 \pm 0.21$ & $1.32 \pm 0.06$ & $1.60 \pm 0.06$ & $1.90 \pm 0.11$ \\
\hline & \multicolumn{8}{|c|}{ Distance to the hole: } \\
\hline & \multicolumn{4}{|c|}{ Peak acceleration [deg.sec ${ }^{-1}$ ] } & \multicolumn{4}{|c|}{ Peak acceleration $\left[\mathrm{m} \cdot \mathrm{s}^{-2}\right]$} \\
\hline Players & $1 \mathrm{~m}$ & $2 \mathrm{~m}$ & $3 \mathrm{~m}$ & $4 \mathrm{~m}$ & $1 \mathrm{~m}$ & $2 \mathrm{~m}$ & $3 \mathrm{~m}$ & $4 \mathrm{~m}$ \\
\hline 1 & $233.92 \pm 31.01$ & $253.86 \pm 36.47$ & $254.22 \pm 21.04$ & $\begin{array}{c}759.60 \pm \\
174.86\end{array}$ & $2.91 \pm 0.23$ & $3.51 \pm 0.43$ & $4.32 \pm 0.58$ & $4.80 \pm 0.89$ \\
\hline 2 & $183.42 \pm 21.34$ & $297.02 \pm 22.48$ & $412.93 \pm 59.63$ & $510.76 \pm 64.97$ & $3.42 \pm 0.33$ & $5.05 \pm 0.46$ & $6.98 \pm 0.46$ & $7.89 \pm 0.54$ \\
\hline 3 & $359.82 \pm 39.13$ & $431.62 \pm 29.17$ & $513.74 \pm 48.49$ & $599.84 \pm 48.31$ & $5.46 \pm 0.39$ & $6.35 \pm 0.68$ & $7.46 \pm 0.49$ & $7.60 \pm 0.38$ \\
\hline 4 & $445.47 \pm 41.60$ & $592.59 \pm 62.65$ & $741.65 \pm 37.09$ & $911.16 \pm 86.92$ & $6.57 \pm 0.53$ & $9.08 \pm 0.82$ & $11.92 \pm 0.62$ & $14.19 \pm 0.92$ \\
\hline 5 & $306.67 \pm 160.49$ & $391.48 \pm 35.04$ & $470.82 \pm 33.17$ & $560.37 \pm 47.48$ & $5.04 \pm 0.37$ & $6.89 \pm 0.49$ & $8.43 \pm 0.62$ & $9.85 \pm 0.69$ \\
\hline 6 & $296.22 \pm 82.48$ & $323.31 \pm 41.79$ & $410.51 \pm 43.77$ & $491.95 \pm 44.68$ & $4.30 \pm 0.77$ & $6.03 \pm 0.60$ & $7.48 \pm 0.67$ & $8.41 \pm 0.67$ \\
\hline 7 & $258.71 \pm 21.73$ & $299.02 \pm 23.48$ & $354.82 \pm 59.63$ & $404.33 \pm 23.71$ & $5.10 \pm 0.43$ & $5.86 \pm 0.34$ & $6.74 \pm 1.16$ & $7.55 \pm 0.42$ \\
\hline 8 & $301.58 \pm 23.55$ & $314.01 \pm 27.58$ & $341.88 \pm 43.28$ & $439.81 \pm 77.19$ & $5.05 \pm 0.38$ & $5.25 \pm 0.41$ & $6.05 \pm 0.84$ & $7.15 \pm 0.60$ \\
\hline 9 & $278.58 \pm 37.94$ & $345.65 \pm 39.32$ & $353.01 \pm 37.97$ & $338.50 \pm 26.41$ & $4.59 \pm 0.56$ & $5.89 \pm 0.45$ & $6.58 \pm 0 . .43$ & $6.96 \pm 0.43$ \\
\hline 10 & $214.71 \pm 34.85$ & $276.60 \pm 22.36$ & $322.96 \pm 22.66$ & $325.77 \pm 25.22$ & $3.81 \pm 0.53$ & $4.98 \pm 0.40$ & $5.98 \pm 0.38$ & $6.28 \pm 0.37$ \\
\hline 11 & $256.37 \pm 98.30$ & $265.34 \pm 32.53$ & $329.29 \pm 58.75$ & $374.31 \pm 38.86$ & $3.91 \pm 1.06$ & $4.11 \pm 0.26$ & $4.91 \pm 0.46$ & $6.11 \pm 0.50$ \\
\hline 12 & $293.33 \pm 27.87$ & $336.39 \pm 28.28$ & $421.11 \pm 34.19$ & $450.74 \pm 41.05$ & $5.08 \pm 0.34$ & $5.97 \pm 0.46$ & $7.20 \pm 0.86$ & $7.59 \pm 0.88$ \\
\hline 13 & $257.28 \pm 33.79$ & $295.15 \pm 36.26$ & $379.48 \pm 42.10$ & $541.09 \pm 61.08$ & $4.88 \pm 0.38$ & $6.19 \pm 0.53$ & $6.99 \pm 0.73$ & $8.82 \pm 1.01$ \\
\hline \multirow[t]{2}{*}{14} & $297.73 \pm 45.42$ & $307.97 \pm 30.92$ & $356.67 \pm 34.07$ & $391.14 \pm 44.04$ & $5.27 \pm 0.73$ & $5.49 \pm 0.36$ & $6.07 \pm 0.45$ & $7.28 \pm 0.46$ \\
\hline & \multicolumn{8}{|c|}{$\begin{array}{l}\text { Distance to the hole: } \\
\text { ct pressure on the ball }[\mathrm{KgF} \text {. }\end{array}$} \\
\hline Players & \multicolumn{2}{|c|}{$1 \mathrm{~m}$} & \multicolumn{2}{|c|}{$2 \mathrm{~m}$} & \multicolumn{2}{|c|}{$3 \mathrm{~m}$} & \multicolumn{2}{|c|}{$4 \mathrm{~m}$} \\
\hline 1 & \multicolumn{2}{|c|}{$1.73 \pm 0.89$} & \multicolumn{2}{|c|}{$2.09 \pm 1.16$} & \multicolumn{2}{|c|}{$1.91 \pm 1.13$} & \multicolumn{2}{|c|}{$1.66 \pm 0.98$} \\
\hline 2 & \multicolumn{2}{|c|}{$1.75 \pm 0.72$} & \multicolumn{2}{|c|}{$234+127$} & 1.9 & 1.44 & & .38 \\
\hline 3 & 0.52 & 0.41 & 0.81 & 0.59 & 1.6 & 1.18 & & .25 \\
\hline 4 & 2.16 & 0.79 & 1.97 & 0.93 & 2.3 & .86 & & .11 \\
\hline 5 & 0.61 & 0.35 & 0.51 & 0.43 & 0.4 & .32 & & .31 \\
\hline 6 & 0.61 & 0.45 & 0.96 & 0.44 & 1.0 & .51 & & .46 \\
\hline 7 & 0.90 & 0.36 & 1.04 & 0.36 & 0.8 & .50 & & .55 \\
\hline 8 & 0.97 & 0.34 & 1.39 & 0.26 & 1.4 & .51 & & .48 \\
\hline 9 & 0.63 & 0.33 & 0.54 & 0.36 & 0.6 & 0.53 & & .59 \\
\hline 10 & 0.56 & 0.28 & 0.82 & 0.22 & 0.8 & .31 & & .45 \\
\hline 11 & 0.80 & 0.33 & 0.81 & 0.33 & 1.0 & .38 & 1.0 & .53 \\
\hline 12 & 1.03 & 0.29 & 1.21 & 0.43 & 1.2 & .47 & & .43 \\
\hline 13 & 0.44 & 0.31 & 0.55 & 0.36 & 0.7 & .33 & & .59 \\
\hline 14 & 0.50 & 0.33 & 0.58 & 0.31 & 0.7 & .37 & 0.8 & .17 \\
\hline
\end{tabular}

Finally, regarding the impact pressure on the ball, players showed higher values within $3 \mathrm{~m}$ of the hole. For the impact velocity a tendency to increase the values obtained 
as the distance to the hole increased was observed. In accordance with the low variation coefficient values obtained for impact velocity $\left(\mathrm{deg} \cdot \mathrm{m}^{-1}\right)$, the players presented a stable motor performance in the four distances. Regarding the impact pressure on the ball $\left(\mathrm{KgF}^{-2}\right), 10$ of the 14 players presented higher values at 2, 3 and $4 \mathrm{~m}$ from the hole when compared to $1 \mathrm{~m}$ (see Table 6 , in attachment). There were statistically significant differences between the four PC $\left(F(3,1677)=6.64, p\right.$-value $\left.=0.001, \eta^{2}=0.01, \pi=0.97\right)$. In this context, participants presented higher values of impact pressure on the ball and with statistical significance between PC1 and PC2 ( $p$-value $=0.009)$, PC3 $(p$-value $=0.001)$ and PC4 $(p$-value $=0.003)$.

\section{Discussion}

The main objective of this study was to analyse the golf-putting performance in skilled golfers at different distances to the hole. We hypothesize that the manipulation of putting distance required the adoption of solutions exclusively adjusted to each player. Regarding the variation of the inter-individual motor performance obtained in the backswing and downswing phases, the variation coefficient ranged between 36\% (PC1) and 23\% (PC4). The results showed a proportional increase in the backswing and downswing duration and maximum duration time as the distance to the hole increased. However, in the remaining phases of putting this tendency of proportionality is not so evident. In this view, golfers continually need to adapt and regulate the putting movement to satisfy the interacting constraints that emerge at a particular point during task performance [2]. Furthermore, the high variability of practice conditions requires constant adaptations to putting distances. As a consequence, each golfer learns to adapt performance under different distances to the hole [2].

The values of the inter-individual variation in the maximum amplitude of the putting (deg and $\mathrm{m}$ ) were relatively low, presenting variation coefficients inferior to $22 \%$ in all the distances. In line with some theoretical considerations [7], as the distance to the hole increased, we observed an increment in the follow-through amplitude. Additionally, the results of the backswing and downswing amplitude indicated statistically significant differences between the four practical conditions. Moreover, the data suggested how golfers continually need to adapt and regulate the putting performance to satisfy the interacting constraints (distances). These trends were evident in the majority of the motor performance presented by the players and in line with the literature [1,16].

The results obtained also showed a longer backswing duration when moving from PC1 to PC3 and to PC4. The follow-through duration remained steady, regardless of the distance to the hole. In contrast with the findings of Dias et al. [2] that were made in a laboratory context, we observed a progressive decrease of the downswing duration as the distance from the hole increased. These results might be justified based on the visual assessment of the player, which is crucial when performing any skill in sports, including the putting which requires striking a ball so it can reach its intended target with the correct speed and trajectory. For instance, the green and other psychological aspects may affect visual processing [8].

The results of backswing duration (ms) indicated that 8 players depicted a tendency to increase the duration of this phase as the distance to the hole increased. Thus, when the distances to the hole increased, the backswing phase took longer. This trend of proportionality was not evident in the downswing duration (ms). We found longer duration backswing between PC1 and PC 3 , and PC1, and PC4. The data also showed that a significant number of players presented slightly higher values in backswing duration (ms) in PC1 compared to the remaining practice conditions (see players 2, 3, 4, 6, 8, 10, 12). However, there were no statistically significant differences between the four PC for this putting phase. The results of impact duration (ms) did not indicate an intra-individual uniform trend of motor performance between the four PC. In this sense, it was evident that most players obtained very high values of standard deviation. Our results encompass relevant variables within the golf-putting performance in skilled golfers at different distances to the hole, namely 
the amplitude, velocity, and acceleration. One way to promote this consists of acquiring data about the motor performance and analysing it to identify and correct any technical inconsistencies [1].

Compared with amplitude and impact velocity $\left(\mathrm{deg} \cdot \mathrm{m}^{-1}\right)$, the variation of peak acceleration values was substantially higher, varying coefficients ranging from $27 \%$ to $34 \%$. There were statistically significant differences between all practice conditions (PC). Regarding the maximum amplitude (deg), the results indicate that most players performed the putting with greater amplitude whenever the distance increased to the hole. However, this was no verified in player 6 from PC1 to PC2. Compared to maximum amplitude (deg), impact velocity and peak velocity $\left(\mathrm{deg} \cdot \mathrm{m}^{-1}\right.$ ) values varied substantially, with $\mathrm{CV}$ values ranging between $27 \%$ and $34 \%$. Follow-through amplitude $(\mathrm{m})$ showed an increase of the values as the distance to the hole increased. This trend was evident in most of the motor performance presented by the players (11 in total). Most players made this movement with greater amplitude when the distance to the hole increased. However, this was not the case for player 3, namely in the PC3 for PC4. Therefore, environmental context and constraints (e.g., distance to the hole) have a significant effect on a player's performance [2]. For instance, the 3- and 4-m distances suggest a strong way to increase the process adjustment. All these factors have a decisive influence on how the golfer will hit the ball and adjust its action [13].

The impact velocity increased significantly with increasing distance to the hole, namely PC1 for PC2, PC2 for PC 3, and PC3 for PC4, but low coefficients were also observed. From an individual perspective our findings suggest that differing strategies may exist where certain golfers may have more "fluid" movement patterns, therefore successfully using movement variability. Consequently, movement variability may be a key determining aspect to the reduction in the variance of the task criterion putter face angle at impact and, therefore, performance [13]. On the other hand, the results of impact duration time did not indicate an intra-individual uniform trend of motor performance between the four PC. Hence, there were no statistically significant differences between the four PC. However, the peak acceleration increased significantly with the distance to the hole. Despite all this, it must also be taken into consideration that the participants' morphologic and functional characteristics, their handicap and the complexity of the task were important to find significant inter-individual differences in the motor performance inferred by the process variables [17].

The results of the speed of impact on the ball $\left(\mathrm{m} . \mathrm{s}^{-1}\right)$ also showed that the majority of players presented higher values as distance increased to the hole. This was not verified only for player 3 from PC 3 to PC4. Additionally, when a performer is in an environment where a high level of accuracy is required, the performer chooses a strategy of "freezing". The ability to use a margin of safety, i.e., to use redundancy of impact speed, would be a more important strategy during actual putting [18]. In this sense, a nonlinear relationship between putting distances and success rates seems to exist, and the success or failure of a golf-putting performance depends on the club-head speed at impact and direction of the clubface [1]. Regarding the process variables of motor performance, professional golfers maintained a high consistency of ball speed and the movement variability was highly individual-specific with different players adopting different performance strategies depending on the distance to the hole [5].

Finally, the peak acceleration $\left(\mathrm{m} . \mathrm{s}^{-2}\right.$ ) results indicate that most players had higher acceleration values in line with the increased distance to the hole. This was not observed for player 7 from PC3 to PC4. Therefore, results also suggested how golfers continually need to adapt the impact duration time and peak acceleration of the putting in order to satisfy the interacting constraints that exist at a particular point during task performance [10]. Thus, vision plays an important role in assisting a golfer to sustain a square putter head stroke, ascertain the best line or path of the ball and optimize gaze control during the putting process [19]. Likewise, professional golfers tend to present high level of lengthways putting 
control from their dominant stance, as a consequence of the several years of training and experience [3].

It is important to understand how coaches can provide better feedback during training regarding the variables relevant to their performance. Another implication of this study is the fact that players may better tune and calibrate their performance with the help of new instruments such as the InPutter, therefore improving their perception of golf-putting performance. Finally, developing a better understanding of the role of performance in golf putting is relevant for coaches and players, as they should allow functional movement behaviours to emerge during practice.

One of the main limitations of this study is the small size of the sample to reach strong conclusions. Furthermore, InPutter should be used in different types of green surfaces in order to verify the intra and inter-individual differences that may emerge from performance. Additionally, our results are specific to the sample that we used and not transferable to other groups of players. Future studies should focus on larger and specific groups of players, such as specific age groups, gender, or expertise level.

\section{Conclusions}

The distance constraints forced the adoption of functional solutions uniquely adjusted to each player. For example, as the distance to the hole significantly changes, so do the informational constraints, shaping how a golfer needs to adapt the motor control relations which regulate performance. Thus, golfers' movements are affected by an interaction with the environment, such as that occurring during perception-action coupling.

It is noticeable that the golfers' functional characteristics may affect the force, acceleration, execution velocity, and amplitude of this movement during the performance. These aspects report to the characteristics and inter-individual profiles that distinguish each player during the putting performance. All these factors have a decisive influence on how the golfer will hit the ball and adjust its performance.

In skilled athletes, emerging actions might be organised to provide adaptive flexibility tailored to their personal constraints and current environmental conditions. This interpretation of putting performance suggests that golfers can adapt and compensate performance by exploring different couplings of information-movement.

On the other hand, individual variability makes the golf putting quite different from player to player and that environmental context and other constraints (distance to the hole) have a significant effect on a player's performance.

Author Contributions: G.D. and M.C. designed the research study; G.D. and M.C. conceived the data collection; P.M., R.M. and F.M. analysis and interpretation the data; G.D., P.M., R.G., V.V., J.G. and M.A.C. performed the drafting the article and/or its critical revision; All authors contributed to editorial changes in the manuscript. All authors have read and agreed to the published version of the manuscript.

Funding: This work is funded by FCT/MCTES through national funds and when applicable cofunded EU funds under the project n. ${ }^{\circ}$ UIDB/50008/2020.

Institutional Review Board Statement: The study was conducted according to the guidelines of the Declaration of Helsinki and approved by the Ethics Committee of Polytechnic Institute of Coimbra (Approval number: N145 CEIP/2021).

Informed Consent Statement: Informed consent was obtained from all subjects involved in the study.

Data Availability Statement: The data presented in this study are available on request by the corresponding author.

Conflicts of Interest: The authors declare no conflict of interest. 


\section{References}

1. Pelz, D. Putting Bible: The Complete Guide to Mastering the Green; Publication Doubleday: New York, NY, USA, 2000.

2. Dias, G.; Couceiro, M.S.; Barreiros, J.; Clemente, F.; Mendes, R.; Martins, F. Distance and slope constraints: Adaptation and variability in golf putting. Mot. Control 2014, 18, 221-243. [CrossRef] [PubMed]

3. Suzuki, T.; Manabe, Y.; Arakawa, H.; Sheahan, J.P.; Okuda, I.; Ichikawa, D. A comparison of stroke distance error from dominant and non-dominant putting stance in professional and novice golfers. Int. J. Golf. Sci. 2019, 7, 1-12.

4. Wang, Y.T.; Su, P.C.; Wu, Y.L.; Jia-Hao, C. Analysis of grip force during golf putting at different distances-Pilot Study. In Proceedings of the 35th Conference of the International Society of Biomechanics in Sports, Cologne, Germany, 4 June 2017; pp. $14-18$.

5. Tucker, C.B.; Anderson, R.; Kenny, I.C. Is outcome related to movement variability in golf? Sports Biomech. 2013, 12, 343-354. [CrossRef] [PubMed]

6. Hume, P.A.; Keogh, J.; Reid, D. The role of biomechanics in maximising distance and accuracy of golf shots. Sports Med. 2005, 35, 429-449. [CrossRef] [PubMed]

7. Delay, D.; Nougier, V.; Orliaguet, J.P.; Coello, Y. Movement control in golf putting. Hum. Mov. Sci. 1997, 16, 597-619. [CrossRef]

8. Dias, G.; Couceiro, M.S. The Science of Golf Putting: A Complete Guide for Researchers, Players and Coaches; Springer Briefs in Applied Sciences and Technology: Berlin/Heidelberg, Germany, 2015.

9. Karlsen, J.; Smith, G.; Nilsson, J. The stroke has only a minor influence on direction consistency in golf putting among elite players. J. Sports Sci. 2008, 26, 243-250. [CrossRef] [PubMed]

10. Keogh, J.W.L.; Hume, P.A. Evidence for biomechanics and motor learning research improving golf performance. Sports Bio-mech 2012, 11, 288-309. [CrossRef] [PubMed]

11. Couceiro, M.S.; Dias, G.; Araújo, A.; Pereira, S. InPutter: Concept and evaluation of an engineered golf putter. S. Afr. J. Res. Sport Phys. Educ. Recreat. 2015, 37, 43-54.

12. Ingeniarius. Available online: http://ingeniarius.pt/inputter (accessed on 6 December 2021).

13. Couceiro, M.S.; Dias, G.; Mendes, R.; Araújo, D. Accuracy of Pattern Detection Methods in the Performance of Golf Putting. J. Mot. Behav. 2013, 45, 37-53. [CrossRef] [PubMed]

14. Pallant, J. SPSS Survival Manual: A Step-by-Step Guide to Data Analysis Using the SPSS Program; Allen \& Unwin: Crows Nest, Australia, 2011.

15. Ferguson, C. An effect size primer: A guide for clinicians and researchers. Prof. Psychol. Res. Pr. 2009, 40, 532-538. [CrossRef]

16. Coello, Y.; Delay, D.; Nougier, V.; Orliaguet, J.P. Temporal control of impact movement: The "time from departure" control hypothesis in golf putting. Int. J. Sport Exerc. Psychol. 2000, 31, 24-46.

17. Lier, W.H.V.; Van der Kamp, J.; Savelsbergh, G.J.P. Perception and Action in Golf Putting: Skill Differences Reflect Calibration. J. Sport Exerc. Psychol. 2011, 33, 349-369. [CrossRef]

18. Hasegawa, Y.; Koyamab, S.; Inomatab, K. Perceived distance during golf putting. Hum. Mov. Sci. 2013, 32, 1226-1238. [CrossRef]

19. DeBroff, B.M. The role of vision in the science and art of the putting stroke in the sport of golf. Adv. Ophthalmol. Vis. Syst. 2018, 8 , 154-157. [CrossRef] 